\title{
ORIGINAL
}

\section{EPIDEMIOLOGÍA DE LAS DESIGUALDADES DEL DESARROLLO DE GÉNERO EN ESPAÑA (1990-2000)}

\section{Mercedes Carrasco-Portiño (1), María Teresa Ruiz-Cantero (1,2,3), Diana Gil-González (1,3) Carlos Álvarez-Dardet Díaz $(1,2,3)$ y Jordi Torrubiano-Domínguez (1).}

(1) Área de Medicina Preventiva y Salud Pública. Universidad de Alicante. España.

(2) CIBER de Epidemiología y Salud Pública (CIBERESP).

(3) Observatorio de Políticas Públicas y Salud, España.

\section{RESUMEN}

Fundamento: El género es un determinante de la salud importante para las Políticas de Salud Pública. Este estudio describe los cambios en las desigualdades del desarrollo de género en España y sus Comunidades Autónomas (CCAA) en la década 1990 y 2000.

Métodos: Estudio ecológico del Índice de Desarrollo Humano (IDH) y el Índice de Desarrollo de Género (IDG) y sus componentes por sexo (educación, ingresos y esperanza de vida al nacer).

Resultados: El IDG de España ha crecido en un 5,05\% en los años 90. Pero, mientras que en 1990 el $51,5 \%$ de la población estaba ubicada por encima del IDG promedio español en 2000 disminuyó al 46,3\%. Asturias: 3,37\%, Cantabria: 3,68\% e Islas Baleares: $3,71 \%$ son las de menor crecimiento; Madrid: $6,46 \%$ y Extremadura: $6,75 \%$ las de mayor. Todas las CCAA. tienen un valor de IDG menor que de IDH. Ambos sexos tuvieron un crecimiento promedio similar en la esperanza de vida (Hombres: 5\% y Mujeres: 4\%) manteniéndose las diferencias, aunque se detectan variaciones según CCAA. En educación, las mujeres mejoraron el doble que los hombres (Hombres: $3 \%$ y Mujeres: 6\%), siendo Cataluña, Aragón, Madrid, Baleares y Galicia las de mayor igualdad. Pese a que las mujeres mejoraron el triple que los hombres en el índice de ingresos (Hombres: $3 \%$ y Mujeres: $9 \%$ ), las diferencias persisten a favor de los hombres.

Conclusiones: Las desigualdades en el desarrollo de género disminuyeron en los 90 en España, aunque la mejora de la educación, ingresos y esperanza de vida se produjo sólo en algunas CCAA, persistiendo diferencias interregionales.

Palabras Clave: Género. Desigualdades. Desarrollo humano. Esperanza de vida. Educación. Renta.

\section{Correspondencia:}

Mercedes Carrasco Portiño

Área de Medicina Preventiva y Salud Pública

Edificio Colegio Mayor

Campus San Vicente del Raspeig. Universidad de Alicante Apartado Postal 99. 03080 Alicante.

Correo electrónico: mercedes.carrasco@ua.es

\section{ABSTRACT}

\section{Gender Development Inequalities Epidemiology in Spain (1990-2000)}

Background: Gender is an important health determinant for public health policies. This study describes the changes in gender development inequalities in Spain and its autonomous regions from 1990 to 2000.

Methods: An ecological study using the Human Development Index (HDI) and the Gender Development Index (GDI) was done. IDG both men and women was analysed according to indexes of education, income and life expectancy at birth.

Results: Although the GDI has had an increase of 5,05\% in the 90 's, $51,5 \%$ of the population was located above the global GDI of Spain in 1990, moreover this number decreased to $46,3 \%$ in 2000 . Gender inequalities have been reduced both at national and regional levels. The regions with the lowest increase were Asturias (3.37\%), Cantabria $(3.68 \%)$ and Baleares Islands $(3.71 \%)$. The regions with the highest increase were Madrid $(6,46 \%)$ and Extremadura $(6,75 \%)$. All the autonomous regions showed a number of GDI lower than the value of HDI. Both sexes achieved similar increase in life expectancy (Men: 5\% and Women: $4 \%$ ). An unequal variation was detected according to the autonomous region (Basque Country; Men: 7\% and Women: 3\%; Madrid; Men:8\% and Women:5\%). Women have improved their educational level in comparison to men (Men: $3 \%$ and Women: $6 \%$ ). In the 90 s, men obtained more income than women, but women improved their situation three times more than men.

Conclusions: Inequalities in Human Development analysed by gender have been reduced in the 90s in Spain. However, the improvement of education, income and life expectancy occurred only in some autonomous regions. This situation shows the differences among Spanish autonomous regions.

Keywords: Gender. Health inequalities. Human Development. Life expectancy. Education. Income. 


\section{INTRODUCCIÓN}

En 1990 el Programa de Naciones Unidas para el Desarrollo (PNUD) puso en marcha una iniciativa orientada a establecer indicadores de desarrollo con el fin de lograr el bienestar mundial. Hasta ese momento se utilizaba como único indicador de desarrollo económico el crecimiento de la renta per capita ${ }^{1}$. Esta nueva perspectiva sugiere agregar un componente de salud y otro de educación a este indicador, creando así el Índice de Desarrollo Humano (IDH). Por lo tanto, el IDH es un índice compuesto que contiene tres variables: la esperanza de vida al nacer, el logro educacional (alfabetización de adultos, y tasa bruta de matriculación primaria, secundaria y terciaria combinada) y el Producto Interior Bruto (PIB) real per cápita (Paridad Poder Adquisitivo (PPA) en dólares) ${ }^{2}$.

El IDH ofrece una aproximación media del nivel de desarrollo considerando más dimensiones y no solo el crecimiento de la economía monetaria, pero no evalúa las diferencias en el desarrollo entre hombres y mujeres por lo que, a partir del interés sobre las desigualdades de género en el desarro1lo, el Informe del Desarrollo Humano de 1995 presentó un nuevo indicador denominado Índice de Desarrollo de Género (IDG). Éste mide el desarrollo en las mismas dimensiones y con las mismas variables que el IDH, pero para el cálculo del IDG se consideran las posibles desigualdades entre mujeres y hombres ${ }^{3}$. La bondad del IDG como indicador de las desigualdades de género en salud se basa en que además de la distribución por sexo en la esperanza de vida, incluye dos importantes determinantes de la salud, el nivel educativo y los ingresos.

La importancia de la sensibilidad de género en el desarrollo ha sido tal que se ha incorporado en el tercer objetivo de desarrollo del milenio (ODM) de la Organización de Naciones Unidas (ONU), el cual se estableció en el año 2000 por 189 países, incluido España. Este objetivo se propone promover la igualdad entre hombres y mujeres y el empoderamiento de las mujeres antes del año 2015. La evaluación de este objetivo se realizará mediante indicadores tales como la proporción de mujeres entre las personas empleadas remuneradas en el sector no agrícola, la proporción de empleos ocupados por mujeres por categoría de puesto de trabajo, y la proporción de escaños ocupados por mujeres en los Parlamentos ${ }^{4}$. En este sentido, desde la Conferencia de Beijing en 1995 y el informe Beijing +5 , la ONU viene destacando la necesidad de adoptar indicadores de género para diagnosticar la situación de las mujeres y hacer políticas públicas consecuentes ${ }^{5,6}$.

Junto con el IDG existen otros índices que permiten evaluar la integración de la mujer en el desarrollo, como el índice de potenciación de género (IPG) (Gender Empowerment Measure) y la brecha de género (IBG) (Gender Gap Index) 7,8 (tabla 1). El IPG incluye entre sus variables la participación de las mujeres en los escaños parlamentarios, en los cargos de responsabilidad pública y en los trabajos técnicos y profesionales, así como la diferencia de los ingresos económicos percibidos por hombres y mujeres ${ }^{7}$. El IBG mide la situación de desigualdad de género en la participación y oportunidades económicas, los logros educativos, empoderamiento político y salud y supervivencia ${ }^{8}$. El informe de 2007 sobre la brecha de género en el mundo establece un ranking de 115 países donde España ocupa el décimo lugar, inmediatamente después de Suecia, Noruega, Finlandia, Islandia, Dinamarca, Nueva Zelanda, Filipinas, Alemania e Irlanda ${ }^{7}$. España se sitúa en la $5^{\text {a }}$ posición en el componente de empoderamiento político, en la $39^{\mathrm{a}}$ del componente de logro educativo, en la $74^{\mathrm{a}}$ respecto al componente salud y esperanza de vida, y en el puesto 84 en el ítem oportunidad y participación económica ${ }^{7}$. 
Tabla 1

Indicadores de las desigualdades del desarrollo de género

\begin{tabular}{|c|c|c|c|c|}
\hline Indice de Desarrollo & Definición & Componentes & $\begin{array}{l}\text { Componentes } \\
\text { del Índice }\end{array}$ & $\begin{array}{c}\text { Variables } \\
\text { de Subíndices }\end{array}$ \\
\hline $\begin{array}{l}\text { Indice Desarrollo de Género (IDG) } \\
\text { (Gender-Related Development Index (GDI)) } \\
\text { Se calcula para } 140 \text { países. } \\
\text { Organismo: Naciones Unidas (PNUD). } \\
\text { Año inicio: } 1995 \\
\text { Publicación: Informe anual de Desarrollo } \\
\text { Humano. } \\
\text { Disponible en: } \\
\text { http://hdr.undp.org/en/media/hdr_1995_en.pdf }\end{array}$ & $\begin{array}{l}\text { IDG: Indicador compues- } \\
\text { to que mide el desarrollo } \\
\text { conseguido de forma } \\
\text { igualmente distribuida } \\
\text { entre hombres }(\mathrm{H}) \text { y } \\
\text { mujeres }(\mathrm{M}) \text {. } \\
\text { Valores: } 0 \text { a } 1^{*} \text {. }\end{array}$ & $\begin{array}{l}\text { Vida Larga y Saludable } \\
\text { Educación } \\
\text { Nivel de Vida Digno }\end{array}$ & $\begin{array}{l}\text { Esperanza de Vida al } \\
\text { Nacer igualmente distri- } \\
\text { buido entre H/M } \\
\text { Educación igualmente } \\
\text { distribuida entre H/M } \\
\text { Ingresos igualmente Dis- } \\
\text { tribuido entre H/M }\end{array}$ & $\begin{array}{l}\text { Esperanza de Vida al } \\
\text { Nacer }(\mathrm{H} / \mathrm{M}) \text {. } \\
\text { 1. Tasa de alfabetización } \\
\text { de adultos }(\mathrm{H} / \mathrm{M}) \text {. } \\
\text { 2. Tasa bruta de matricu- } \\
\text { lación } 1^{\mathrm{a}}, 2^{\mathrm{a}} \text { y superior } \\
\text { combinada }(\mathrm{H} / \mathrm{M}) \text {. } \\
\text { Cálculo de Ingresos per- } \\
\text { cibidos }(\mathrm{H} / \mathrm{M}) \text {. }\end{array}$ \\
\hline $\begin{array}{l}\text { Índice de Potenciación de Género (IPG) } \\
\text { (Gender Empowerment Measure (GEM)) } \\
\text { Se calcula para } 80 \text { países. } \\
\text { Organismo: Naciones Unidas (PNUD). } \\
\text { Año inicio: } 1995 \\
\text { Publicación: Informe anual de Desarrollo } \\
\text { Humano. } \\
\text { Disponible en: } \\
\text { http://hdr.undp.org/en/media/hdr_1995_en.pdf }\end{array}$ & $\begin{array}{l}\text { IPG: Indicador compues- } \\
\text { to que mide progreso de } \\
\text { igualmente de hombres } \\
\text { (H) y mujeres }(\mathrm{M}) \text {, en } \\
\text { esferas fundamentales de } \\
\text { la participación y la adop- } \\
\text { ción de decisiones econó- } \\
\text { micas y políticas. } \\
\text { Valores: } 0 \text { a } 1^{*} \text {. }\end{array}$ & $\begin{array}{l}\text { Participación Política y } \\
\text { poder de decisión } \\
\text { Participación económica } \\
\text { y poder de decisión } \\
\text { Control de los recursos } \\
\text { económicos }\end{array}$ & $\begin{array}{l}\text { \% de representación par- } \\
\text { lamentaria igualmente } \\
\text { distribuido entre H/M. } \\
\% \text { de participación eco- } \\
\text { nómica igualmente distri- } \\
\text { buido entre H/M. } \\
\% \text { de ingresos igualmen- } \\
\text { te distribuido entre H/M. }\end{array}$ & $\begin{array}{l}\text { Proporción de H/M en } \\
\text { escaños parlamentarios. } \\
\text { 1. Participación de H/M; } \\
\text { como legisladores, altos } \\
\text { funcionarios o directivos. } \\
\text { 2. Participación de H/M } \\
\text { como profesionales y téc- } \\
\text { nicos. } \\
\text { Cálculo de ingresos per- } \\
\text { cibidos por H/M. }\end{array}$ \\
\hline $\begin{array}{l}\text { Indice de la Brecha de Género (IBG) } \\
\text { (Gender Gap Index (GGI)) } \\
\text { Se calcula para } 115 \text { países } \\
\text { Organismo: Foro Económico Mundial. } \\
\text { Año inicio: } 2005 \\
\text { Publicación: Informe anual Global de la Bre- } \\
\text { cha de Género. } \\
\text { Disponible } \\
\text { http://www.weforum.org/en/initiatives/gcp/G } \\
\text { ender\%20Gap/index.htm }\end{array}$ & $\begin{array}{l}\text { IBG: Indicador compues- } \\
\text { to que mide el desarrollo } \\
\text { conseguido de forma } \\
\text { igualmente distribuida } \\
\text { entre hombres }(\mathrm{H}) \text { y } \\
\text { mujeres }(\mathrm{M}) \text { en aspectos } \\
\text { económicos, legales y } \\
\text { sociales de la brecha de } \\
\text { género en cada país. } \\
\text { Valores: } 0 \text { a } 1^{*} \text {. }\end{array}$ & $\begin{array}{l}\text { Participación y oportuni- } \\
\text { dades económicas. } \\
\text { Logro educativo. } \\
\text { Participación política. } \\
\text { Salud y supervivencia. }\end{array}$ & $\begin{array}{l}\text { Índice de participación y } \\
\text { oportunidad económica. } \\
\text { Índice de logros educativos } \\
\text { Índice de participación } \\
\text { política } \\
\text { Índice de salud y supervi- } \\
\text { vencia }\end{array}$ & $\begin{array}{l}\text { 1. Participación de muje- } \\
\text { res en la fuerza de trabajo } \\
\text { 2. Igualdad de salarios } \\
\text { entre H/M } \\
\text { 3. Participación de H/M } \\
\text { en altos cargos, gerentes } \\
\text { o directivos } \\
\text { 4. Participación de H/M } \\
\text { en trabajos profesionales } \\
\text { y técnicos, y } \\
\text { 5. \% de H/M desemplea- } \\
\text { dos. } \\
\text { 1. Índice de alfabetiza- } \\
\text { ción de H/M } \\
\text { 2. H/M inscritos en edu- } \\
\text { cación } 1^{\mathrm{a}}, 2^{\mathrm{a}} \text {, superior } \\
\text { 3. Promedio de años } \\
\text { escolarizados de H/M } \\
\text { 1. \% de H/M con escaños } \\
\text { parlamentarios } \\
\text { 2. \% de H/M liderando } \\
\text { ministerios, } \\
\text { 3. \% de H/M jefatura de } \\
\text { gobiernos autonómicos. } \\
\text { 1. Esperanza de Vida la } \\
\text { Nacer de H/M } \\
\text { 2. \% de H/M durante el } \\
\text { nacimiento. }\end{array}$ \\
\hline
\end{tabular}

* Los valores cercanos a 1 indican menos desigualdad y los valores cercanos a 0 indican la presencia de más desigualdad.

El status social de las mujeres es importante porque se han detectado consecuencias medibles para la salud de ambos $\operatorname{sexos}^{9,10}$. Varía según áreas geopolíticas incluso dentro del propio país. Pero, debi- do a la falta de información de los determinantes de contexto social que se relacionan con los problemas de salud, los riesgos para la salud de las mujeres suelen quedar fuera de contexto y despolitizados. Su 
principal consecuencia es que la posibilidad de desarrollo de políticas y estrategias efectivas para mejorar la situación social de las mujeres y su repercusión en la salud queda limitada.

El IDG permite hacer visibles la evolución dinámica de las desigualdades de género de un país, provincia o región ${ }^{3}$. Sin embargo, pese a las bondades de esta medida, es incipiente la producción científica que considera al IDG, o a algunos de sus componentes, cómo un indicador central de sus estudios ${ }^{11-14}$. En este sentido hay que destacar la existencia de algunas iniciativas que relacionan el IDG con fenómenos sociales que tienen impacto en la salud de la mujeres ${ }^{15}$, como es la violencia por parte de sus parejas ${ }^{13}$.

En investigaciones sobre desarrollo humano realizadas en España se observa un abordaje similar al que se está realizando en el ámbito internacional, básicamente centrado en un enfoque económico, pues hacen hincapié en las diferencias en el ingreso per capita por comunidades autónomas (CCAA) y provincias, pero dejando fuera la desagregación de la población por sexo u obviando un enfoque integral ${ }^{16-18}$. De forma excepcional se han ofrecido alternativas metodológicas para medir las desigualdades de género ${ }^{19}$, aunque recientemente se ha publicado un documento que proporciona pautas para el estudio de las desigualdades de género en salud ${ }^{20}$. No obstante, se necesita más conocimiento sobre otros componentes que evalúen el desarrollo de género, especialmente en España, donde en los últimos 30 años se han desarrollado procesos de descentralización política relevantes, que han derivado en la transferencia a la mayoría a los gobiernos de las CCAA.

A partir de los únicos datos existentes sobre el IDG en España se pretende describir los cambios en las desigualdades del desarrollo de género en el ámbito nacional y de sus Comunidades Autónomas en la década 1990 y 2000.

Los objetivos específicos de este trabajo son: comparar el recorrido del Índice desarrollo humano e Índice de desarrollo de género por CCAA entre los años 1990 y 2000, describir la trayectoria del índice de desarrollo de género por CCAA entre los años 1990 y 2000, e identificar el grado de consecución de la equidad entre hombres y mujeres en relación con el curso de los subíndices que componen el Índice de desarrollo de género (esperanza de vida al nacer, educación e ingresos) por CCAA entre los años 1990 y 2000.

\section{MATERIAL Y MÉTODOS}

Diseño: Estudio ecológico del IDG y de sus componentes (índice de educación, de ingresos y de esperanza de vida en hombres y mujeres), del IDG por sexo y del IDH, en España y sus CCAA. en los años 1990 y 2000.

Unidad de análisis: Las unidades son las 17 CCAA (Andalucía, Aragón, Asturias, Baleares, Canarias, Cantabria, Castilla La Mancha, Castilla y León, Cataluña, Comunidad Valenciana, Extremadura, Galicia, La Rioja, Madrid, Murcia, Navarra, País Vasco). En la tabla 2 se puede observar la población por sexo de cada Comunidad Autónoma según los Censos de Población 1991 y 2001, que son los años más cercanos a los estudiados.

Fuentes de Información: Los valores de los años 1990 y 2000 del IDG y sus subíndices, y del IDH, son datos secundarios obtenidos del Informe sobre Capital Humano en España ${ }^{21}$.

Indicadores: Índice de Desarrollo Humano (IDH), Índice de Desarrollo de Género (IDG) y los subíndices que lo 
Tabla 2

Población según sexo de las 17 Comunidades Autónomas Españolas en los años 1991-2001

\begin{tabular}{|l|r|r|r|r|}
\hline \multirow{2}{*}{ CCAA } & \multicolumn{2}{|c|}{ Población 1191 } & \multicolumn{2}{c|}{ Población 2001 } \\
\cline { 2 - 5 } & Mujeres & Hombres & Mujeres & Hombres \\
\hline Andalucía & 3.524 .231 & 3.416 .291 & 3.735 .492 & 3.622 .066 \\
\hline Aragón & 602.247 & 585.570 & 609.369 & 594.846 \\
\hline Asturias & 566.149 & 527.788 & 554.003 & 508.995 \\
\hline Baleares & 360.508 & 348.630 & 424.355 & 417.314 \\
\hline Canarias & 752.305 & 741.479 & 851.511 & 842.966 \\
\hline Cantabria & 269.521 & 257.805 & 274.545 & 206.586 \\
\hline Castilla-La Mancha & 835.657 & 822.789 & 884.966 & 875.550 \\
\hline Castilla y León & 1.286 .395 & 1.259 .531 & 1.246 .600 & 1.209 .874 \\
\hline Cataluña & 3.096 .552 & 2.962 .942 & 3.236 .579 & 3.106 .531 \\
\hline Comunidad Valenciana & 1.969 .017 & 1.888 .217 & 2.115 .792 & 2.046 .984 \\
\hline Extremadura & 536.878 & 524.974 & 534.142 & 524.361 \\
\hline Galicia & 1.413 .770 & 1.317 .899 & 1.401 .502 & 1.294 .378 \\
\hline La Rioja & 132.934 & 130.500 & 138.875 & 137.827 \\
\hline Madrid & 2.563 .855 & 2.383 .700 & 2.813 .638 & 2.609 .746 \\
\hline Murcia & 531.074 & 514.527 & 600.381 & 597.265 \\
\hline Navarra & 261.599 & 257.678 & 279.200 & 276.629 \\
\hline País Vasco & 1.070 .061 & 1.033 .980 & 1.064 .706 & 1.017 .881 \\
\hline
\end{tabular}

Fuente: Instituto Nacional de Estadística. Censo de Población 1991 y 2001.

componen (Índice de Esperanza de Vida al Nacer de Mujeres y Hombres, Índice de Educación de Mujeres y Hombres e Índice de Ingresos de Mujeres y Hombres). Tanto el IDG como el IDH se expresan con un valor, que se construye con la media aritmética de idénticos subíndices (esperanza de vida, educación, ingresos), a los que se les asigna igual peso. La única diferencia entre el IDH y el IDG es que el primero valora las desigualdades de la población en conjunto, y el segundo, después de calcular los subíndices para hombres y mujeres por separado, calcula si esta distribución entre sexos está distribuida igualmente o, lo que es lo mismo, considera las diferencias entre hombres y mujeres $^{21}$. Los valores del IDG como índice global oscilan entre 0 y 1 , indicando menor desigualdad al acercarse a 1 y mayor desigualdad al acercarse a 0 . A su vez cada subíndice del IDG (esperanza de vida, educación, ingresos para hombres y mujeres) también tiene valores que fluctú- an entre el 0 (mínimo desarrollo) y 1 (máximo desarrollo). El progreso en el IDG entre 1990 y 2000 se produce por modificaciones en sus subíndices (esperanza de vida, la educación y los ingresos) tanto en hombres como en mujeres, atendiendo a diversas combinaciones posibles pues, en concreto, lo que mide en cada CCAA es la distancia de los valores entre ambos sexos, sin distinguir si mejoran hombres o mujeres.

En el Informe sobre el Capital Humano en España se puede observar la composición de los subíndices del IDG:

a) Índice educación en hombres y mujeres. Constituido por el nivel de instrucción de hombres y mujeres, con un componente que corresponde las $2 / 3$ partes a la tasa de alfabetización de adultos (hombres y mujeres) y $1 / 3$ parte a la tasa bruta de matriculación combinada (primaria, secundaria y terciaria). Su cálculo es directo dado que estas 
variables ya están normalizadas ${ }^{21}$. En concreto, calcula el valor del desarrollo en la educación de hombres y mujeres, permitiendo determinar la distancia entre ambos sexos. La fuente de información es la Encuesta de Población Activa del Instituto Nacional de Estadística.

b) Índice de ingresos en hombres y mujeres. Constituido por el salario medio no agrícola para hombres y mujeres, el cociente entre el salario medio de hombres y mujeres, las tasas de actividad para hombres y mujeres y finalmente, el PIB real per cápita ajustado. El ajuste del ingreso se realiza mediante una transformación logarítmica 21. En concreto, calcula el valor del desarrollo en los ingresos de hombres y mujeres, permitiendo determinar la distancia entre ambos sexos. Las fuentes de información son la Contabilidad Nacional Trimestral y la Contabilidad Regional de España del Instituto Nacional de Estadística.

c) Índice de esperanza de vida al nacer en hombres y mujeres. Calcula el valor del desarrollo en la supervivencia de hombres y mujeres, permitiendo determinar la distancia entre ambos sexos. Su cálculo es directo dado que los valores están normaliza$\operatorname{dos}^{21}$. Las fuentes de información son las Tablas de Mortalidad del Instituto Nacional de Estadística.

Análisis de datos: Para comparar el recorrido del IDH e IDG por CCAA entre los años 1990 y 2000, se realizó una ordenación decreciente de todos los valores de ambos índices por CCAA y el valor global de España, tanto para el año 1990 como 2000, tomando como referencia los datos de IDG del año 1990.

En segundo lugar, la siguiente fórmula ejemplifica para el caso de Andalucía el análisis de los datos realizado con el fin de describir la trayectoria del IDG (1990 y 2000), que es también lo que se ha hecho para calcular la trayectoria del IDG en el resto de las CCAA y para el valor global de España (1990 y 2000). En concreto, al valor del IDG del año 2000 (de las CCAA y España) se le sustrajo el valor del IDG del año 1990 (de las CCAA y España), dividiéndose el resultado por el valor del IDG del año de referencia (1990), y multiplicándose por 100 para obtener el valor porcentual:

Valor IDG de Andalucía del año 2000 - valor IDG de Andalucía del año 1990 x 100

Valor IDG de Andalucía del año 1990 (año de referencia)

En tercer lugar, con el fin de identificar el grado de consecución de la equidad entre hombres y mujeres en los subíndices que componen el IDG por CCAA en los años 1990 y 2000, se calculó la distancia que existe en los valores de estos subíndices entre hombres y mujeres. Para ello, al valor mayor se le sustrajo el menor en cada una de las CCAA y España, y se ordenaron de forma ascendente, tomando como referencia los datos del año 1990.

Además, se calculó el incremento porcentual del valor de cada subíndice (esperanza de vida, educación e ingresos) para las CCAA y para el valor global del país de hombres y mujeres, respectivamente, en los años 1990 y 2000. La siguiente fórmula ejemplifica para el caso de Madrid el cálculo del índice de ingresos de las mujeres (1990 y 2000), lo cual se realizó para el resto de las CCAA y el valor global de España:

Índice Ingresos de Mujeres de Madrid (2000) - Índice Ingresos de Mujeres de Madrid (1990) $x 100$ Índice Ingreso de Mujeres de Madrid para el año 1990 (año de referencia

Para los subíndices esperanza de vida al nacer y educación en hombres y mujeres se realizaron los mismos cálculos.

El análisis de los datos se realizó con el programa Excel y el paquete estadístico SPSS $11.5^{22}$. 


\section{RESULTADOS}

Desde 1990 a 2000 se produjo una variación positiva del desarrollo de género (IDG) en todas las CCAA en la medida en que se han estrechado las distancias entre los valores de hombres y mujeres en los subíndices de esperanza de vida, educación e ingresos que componen este índice. De la misma forma en la figura 1 se puede observar que el indicador de desigualdad en el desarrollo territorial del país (IDH) ha mejorado durante la década de los noventa. Sin embargo, la brecha entre las desigualdades de género es mayor que la desigualdad en el desarrollo territorial, pues se aleja más de la unidad. En el año 1990 se observa que 10 de las CCAA estaban ubicadas sobre el IDG de España $(0,852)$ y diez años después sólo 8 de las CCAA se encuentran sobre el valor global del país (0,895). Las CCAA que han descendido son Asturias $(0,890)$ y las Islas Baleares $(0,894)$ al experimentar el menor incremento de su IDG entre los años 1990 y 2000. En el caso de Asturias se explica porque el resto de las CCAA. ha mejorado más la igualdad entre hombres y mujeres (figuras 3-5). Lo mismo sucede en Baleares pese a que se han estrechado las distancias en los valores de los tres subíndices entre ambos sexos, en el año 2000 respecto a 1990 (Figuras 3-5).

En la figura 2 se observan las diferencias de crecimiento en términos porcentuales del IDG entre las 17 CCAA, así como el crecimiento del IDG global en España, que fue del 5,05\% en 2000 respecto a 1990 (Figura 2). Sin embargo, mientras que en 1990 el $51,5 \%$ de la población estaba ubicada por encima del IDG global español en 2000 disminuyó al $46,3 \%$ (Tabla 2). El incremento porcentual del IDG oscila entre el 3,37\% (Asturias) y el $6,73 \%$ (Extremadura), siendo esta última la que presentaba más desigualdades de género del país, tanto a comienzos de la década de los noventa como en el 2000. Lo que explica el crecimiento en el IDG de Extremadura es que se ha producido el mayor acortamiento de la distancia del país en el valor del índice

Figura 1

Distribución del IDG* e IDH)** de España y sus CC.AA. 1990 y 2000

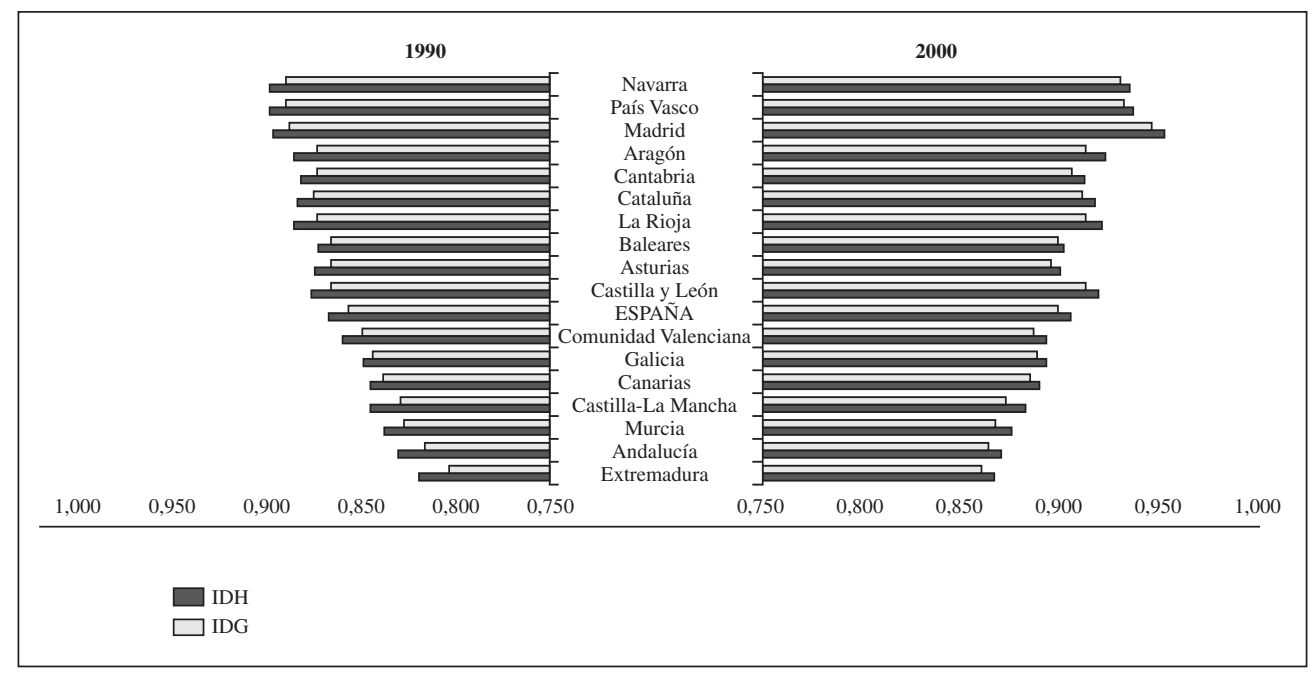

Valores Índice Desarrollo de Género* (IDG) e Índice Desarrollo Humano** (IDH) [0 = más desigualdad y $1=$ menos desigualdad $]$ 
Figura 2

Crecimiento (\%) del IDG de España y sus CC.AA.

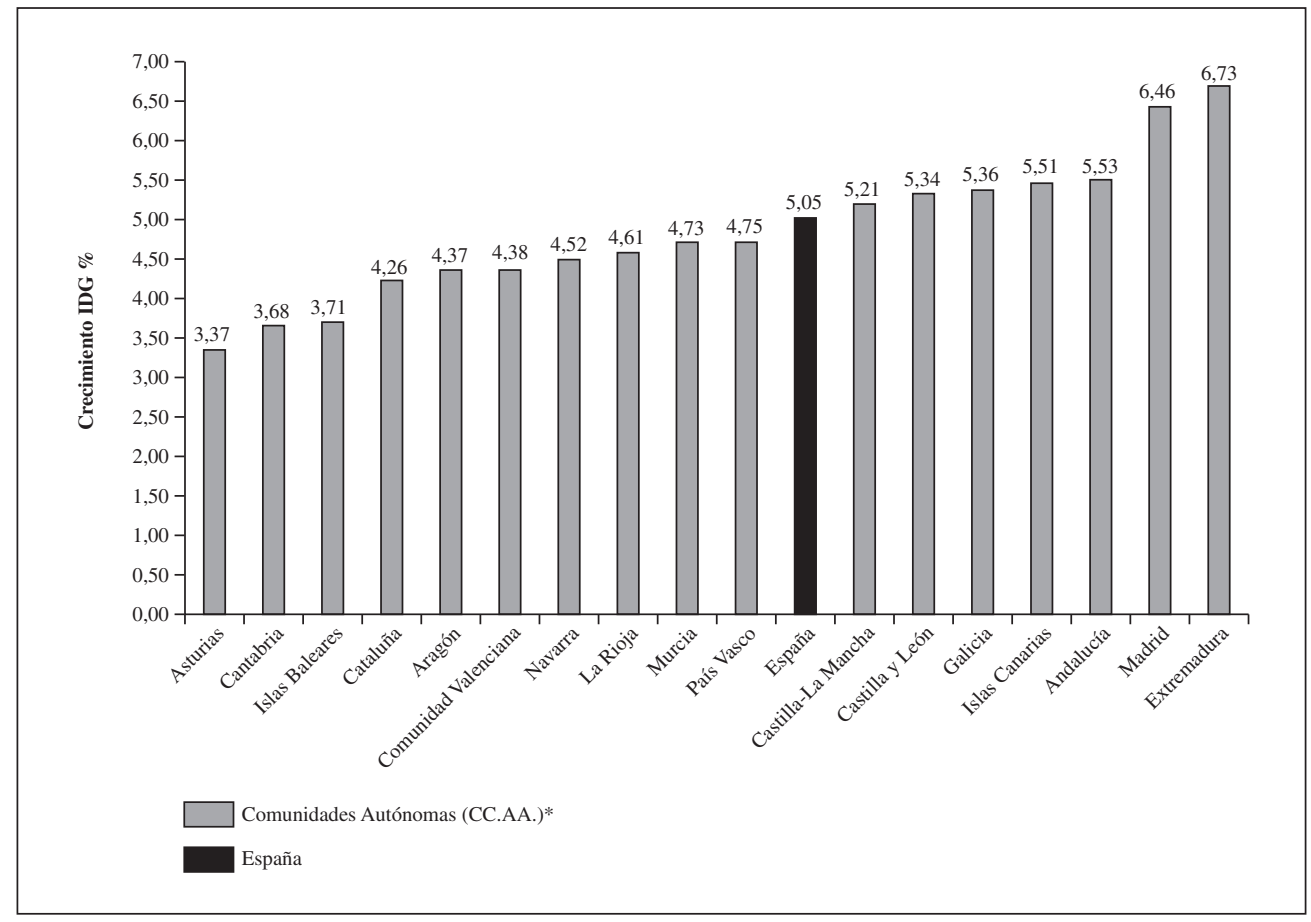

* En 2000 respecto a 1990.

de ingresos entre ambos sexos, así como se ha estrechado la distancia en el valor del índice de educación (figuras 3-5 y tabla 3).

Diez Comunidades Autónomas (59\%) experimentan un valor porcentual inferior al valor global del IDG de España $(5,05 \%)$ (figura 2). Destacan Asturias (3,37\%) e Islas Baleares $(3,71 \%)$ con las cifras mas bajas del crecimiento del IDG que, como se ha comentado, han experimentado el menor crecimiento de todas las CCAA. Y también Cantabria $(3,68 \%)$, donde el incremento por debajo del IDG global del país tiene la misma explicación que para Asturias y las Islas Baleares, esto es, que el resto de las CCAA ha mejorado la igualdad entre ambos sexos más que estas Comunidades. Pero también se debe al importante aumen- to de las distancias de los valores del índice de educación entre ambos sexos a expensas del incremento en las mujeres (figura 3-5).

Las CCAA que han disminuido sus desigualdades de género en mayor medida son Extremadura $(6,73 \%$ ya comentado) y Madrid (6,46\%) (figura 2). El caso de Madrid, que presenta los valores más altos de IDH e IDG de España en 1990 y 2000, se explica por el marcado descenso de las distancias en los valores de los índices de educación y esperanza de vida entre hombres y mujeres (figura 3 y 4) y por el descenso, aunque más leve, de las distancias entre los valores del índice de ingresos (figura 5).

En cuanto al índice de esperanza de vida en la figura 3 se advierte que los hombres 
estaban en 1990 en desventaja respecto a las mujeres. También se observa que Castilla La Mancha es la CCAA que presenta las menores distancias entre hombres y mujeres en este índice, tanto en 1990 como en 2000. Este hallazgo no quiere decir que sus habitantes tengan las mayores cifras de esperanza de vida del país, aunque para los hombres sea el caso. Por el contrario, País Vasco y Asturias presentaban en 1990 las mayores distancias entre ambos sexos, lo que implica que eran las CCAA. con mayores desigualdades de género en esperanza de vida. Esto ocurría por que la esperanza de vida de las mujeres era de las mejores, en especial en el País Vasco, lo que no sucedía en el caso de los hombres. En 2000, pese a que el índice de esperanza de vida mejora en Asturias como sucede en el resto del país, sigue manteniendo la posición de mayor desigualdad de género.

Debido a que en ambos colectivos se ha producido un crecimiento global de la espe- ranza de vida en el país casi similar (mujeres: 4\%; hombres: 5\%), en el año 2000 respecto a 1990, aún se mantienen las diferencias (tabla 3). En este período destaca que en 6 de las CCAA el crecimiento de la esperanza de vida de las mujeres se ha producido por encima del valor global del país, y lo mismo ha sucedido para los hombres en 5 CCAA. Además, considerando todas las CCAA, donde los hombres han mejorado más su esperanza de vida ha sido, porcentualmente, en Madrid (8\%). Para el caso de las mujeres, éstas han aumentado más su esperanza de vida ha sido también en Madrid, junto con Cantabria, Castilla y León, Extremadura, Galicia y Navarra (5\%) (tabla 3).

La figura 4 muestra las diferencias en la distribución entre hombres y mujeres en el índice de educación. En el año 1990 destaca La Rioja como la única Comunidad que presentaba igualdad absoluta en los valores del índice de educación en

Tabla 3

Crecimiento de los Componentes del IDG (\%) en hombres y mujeres en España y sus CC.AA. 1990 y 2000

\begin{tabular}{|c|c|c|c|c|c|c|}
\hline \multirow{2}{*}{ Comunidades Autónomas } & \multicolumn{2}{|c|}{$\begin{array}{l}\text { Crecimiento del Índice } \\
\text { Esperanza de Vida (\%) }\end{array}$} & \multicolumn{2}{|c|}{$\begin{array}{l}\text { Crecimiento del Índice } \\
\text { de Educación }(\%)\end{array}$} & \multicolumn{2}{|c|}{$\begin{array}{l}\text { Crecimiento del Índice } \\
\text { de Ingresos (\%) }\end{array}$} \\
\hline & Hombres & Mujeres & Hombres & Mujeres & Hombres & Mujeres \\
\hline Andalucía & 4 & 3 & 4 & 9 & 2 & 11 \\
\hline Aragón & 3 & 3 & 4 & 6 & 3 & 7 \\
\hline Asturias & 5 & 4 & 0,1 & 3 & 3 & 6 \\
\hline Baleares & 6 & 4 & 1 & 3 & 2 & 6 \\
\hline Canarias & 4 & 3 & 5 & 8 & 3 & 11 \\
\hline Cantabria & 5 & 5 & 0,1 & 2 & 4 & 6 \\
\hline Castilla-La Mancha & 5 & 4 & 4 & 7 & 2 & 9 \\
\hline Castilla y León & 4 & 5 & 4 & 5 & 4 & 10 \\
\hline Cataluña & 5 & 3 & 2 & 4 & 3 & 8 \\
\hline Comunidad Valenciana & 4 & 3 & 3 & 5 & 3 & 8 \\
\hline Extremadura & 5 & 5 & 7 & 11 & 1 & 11 \\
\hline Galicia & 6 & 5 & 3 & 7 & 4 & 7 \\
\hline La Rioja & 6 & 4 & 1 & 4 & 3 & 10 \\
\hline Madrid & 8 & 5 & 5 & 6 & 5 & 10 \\
\hline Murcia & 3 & 3 & 5 & 11 & 2 & 5 \\
\hline Navarra & 4 & 5 & 3 & 3 & 4 & 8 \\
\hline País Vasco & 7 & 3 & 2 & 3 & 3 & 11 \\
\hline España & 5 & 4 & 3 & 6 & 3 & 9 \\
\hline
\end{tabular}




\section{Figura 3}

Distribución del Índice de Esperanza de vida al nacer de hombres y mujeres en España, y sus CC.AA. 1990 y 2000

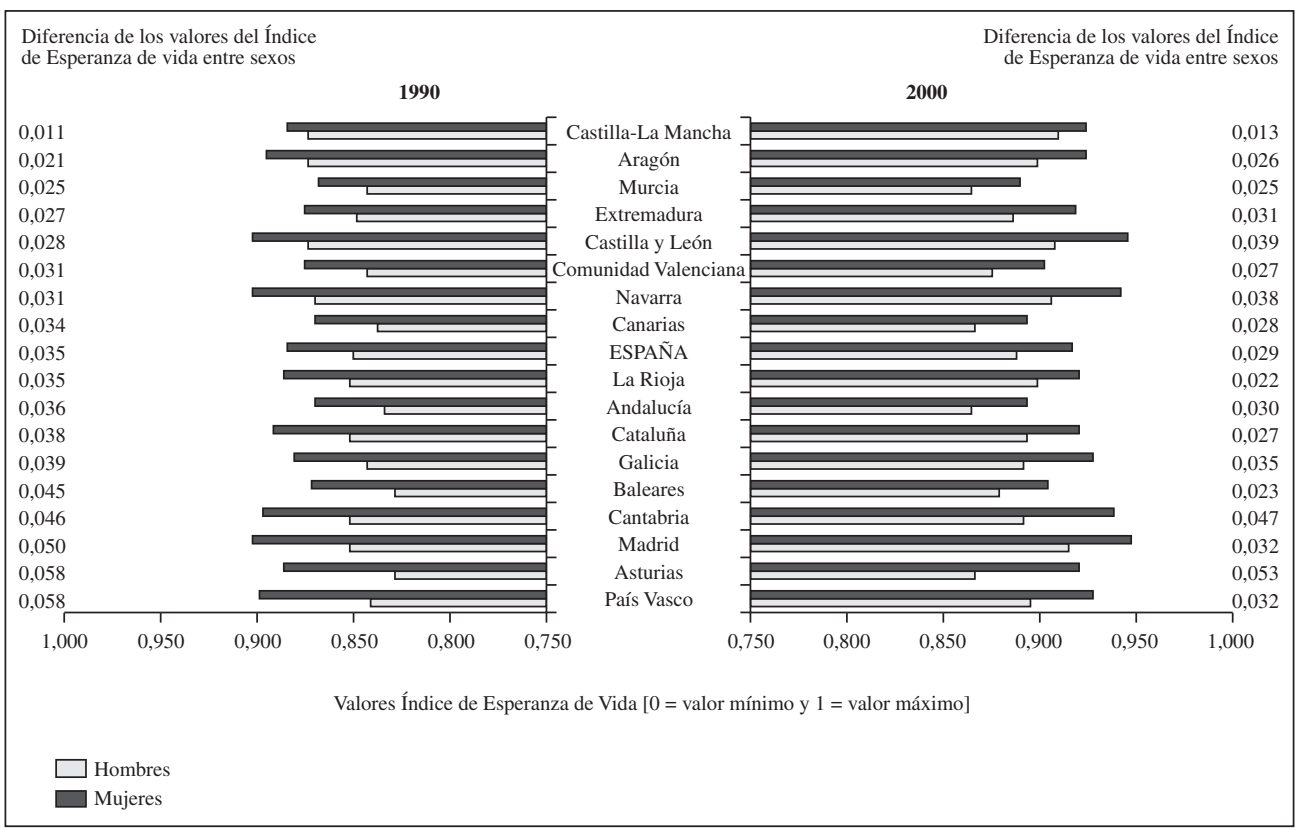

Figura 4

Distribución del Índice de Educación de hombres y mujeres en España, y sus CC.AA. 1990 y 2000

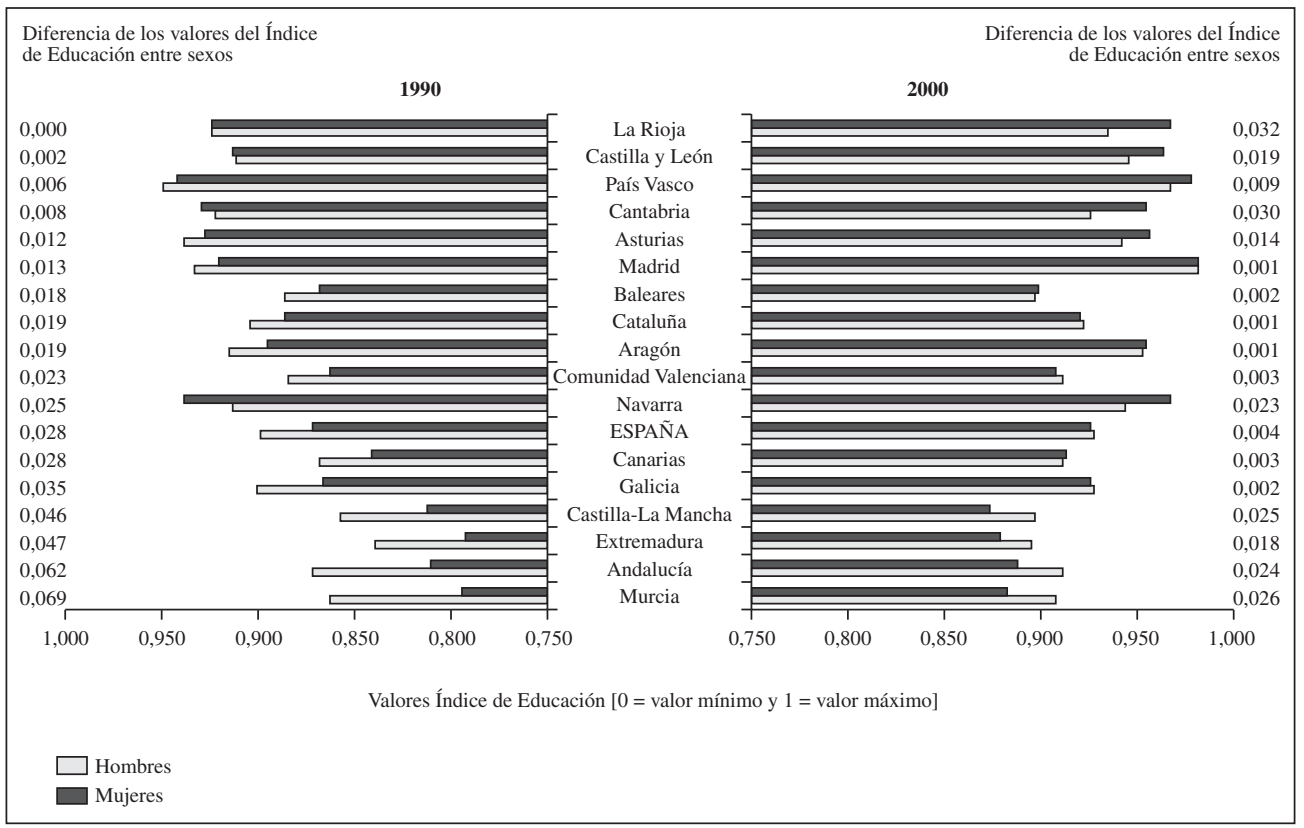


ambos sexos, aunque según este índice no era la CCAA más desarrollada. Sin embargo, sorprendentemente en el año 2000 era la CCAA que presentó la mayor distancia entre hombres y mujeres, pues las mujeres incrementan más sus valores distanciándose de los hombres. Además, en el año 2000, Cataluña, Aragón, Madrid, Baleares y Galicia son las CCAA que alcanzaron los valores más igualitarios entre ambos sexos en el índice de educación, siendo Madrid la Comunidad más desarrollada.

Las mujeres comenzaron la década estudiada muy por debajo del índice de educación de los hombres (figura 4), pero la tabla 3 nos muestra que a nivel global de país en el año 2000 este indicador creció porcentualmente el doble en las mujeres que los hombres en (mujeres: 6\%; hombres: $3 \%$ ). Las CCAA de mayor crecimiento porcentual en el índice de educación para el caso de las mujeres han sido
Extremadura, Murcia, Andalucía y las Islas Canarias, mientras que Extremadura es la CCAA de mayor crecimiento para los hombres. Por el contrario, Cantabria es la CCAA. de menor crecimiento en educación en las mujeres, mientras que esta misma CCAA junto con Asturias son las de menor crecimiento en el índice de educación en los hombres, las cuales apenas han crecido en 10 años.

En la figura 5 se observa que existen grandes diferencias entre los ingresos de hombres y mujeres, en detrimento de estas últimas, aunque ambos sexos mejoraron este componente en 2000 respecto a 1990. Galicia es la CCAA en la que menor distancia había en el índice de ingresos entre hombres y mujeres en 1990, aumentando la distancia en el año 2000. No obstante, ni en 1990 ni en el año 2000 era la Comunidad con valores más desarrollados en el índice de ingresos. Baleares, situada en segunda posición después de Galicia en 1990, en el

Figura 5

Distribución del Índice de Ingresos de hombres y mujeres en España, y sus CC.AA. 1990 y 2000

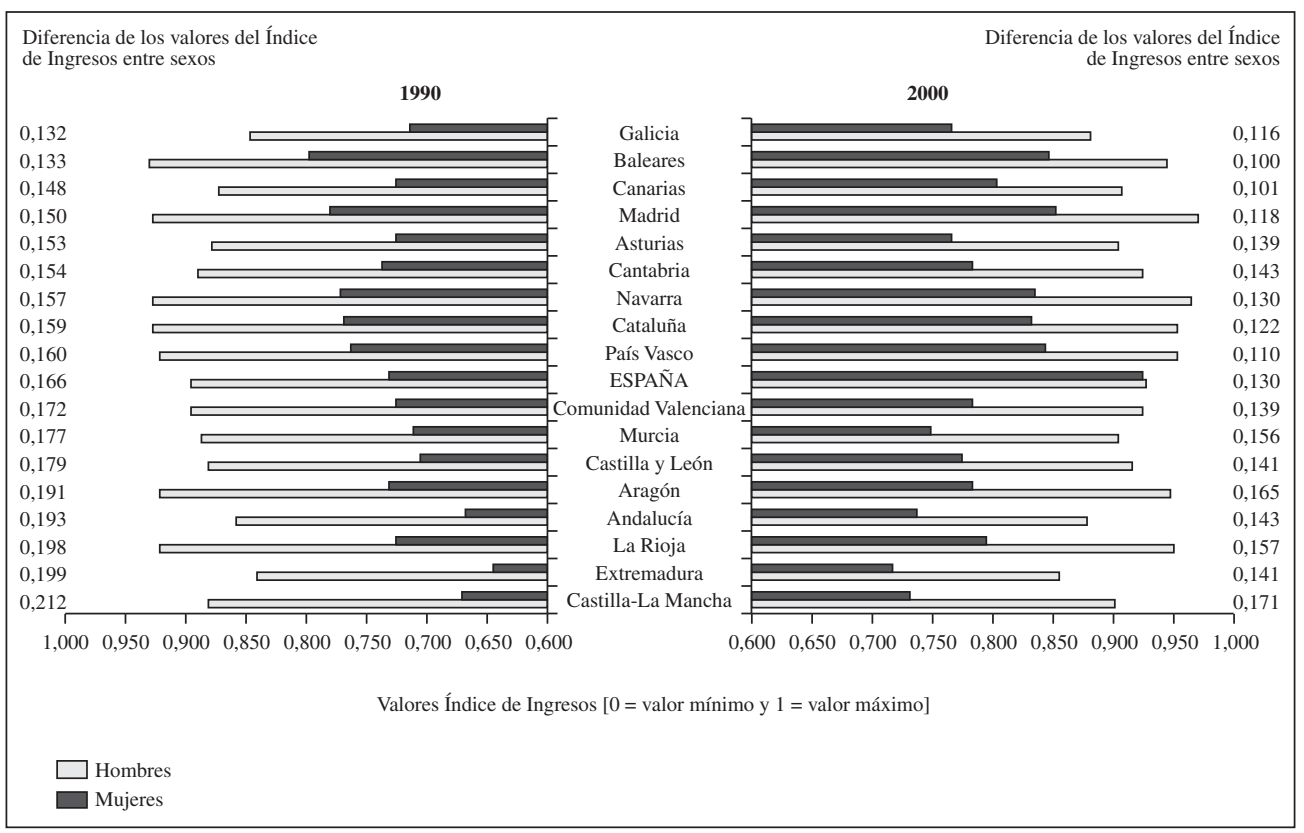


2000 se posicionó en primer lugar en cuanto a menor distancia en los ingresos entre ambos sexos. En Baleares los hombres presentaban los valores del índice de ingresos más desarrollados del país en 1990, mientras que entre las mujeres, son las de esta CCAA. las que ocupan esta posición en 2000. Por el contrario, Castilla La Mancha es la CCAA que presenta la mayor distancia entre hombres y mujeres en el índice de ingresos en 1990 y 2000.

Las mujeres han crecido 3 veces más que los hombres en el índice de ingresos a nivel global del país (mujeres: 9\%; hombres: $3 \%$ ), aunque existen diferencias en todas las CCAA. (tabla 3). Destacan con el mayor crecimiento las mujeres de Andalucía, Canarias, Extremadura y el País Vasco, y Madrid como la de mayor crecimiento de los hombres. Por el contrario, son los hombres de Extremadura los de menor crecimiento junto con las mujeres de Murcia.

\section{DISCUSIÓN}

En España, durante la última década del siglo XX se redujeron las desigualdades en el denominado desarrollo humano y las de género. Todas las CCAA han mejorado estos índices. No obstante, las desigualdades en el desarrollo de género aún persisten a lo largo del territorio español, debido a que se mantienen las diferencias entre sexos en educación e ingresos en perjuicio de las mujeres, y en esperanza de vida en perjuicio de los hombres. Los efectos de esta situación en las desigualdades en salud presente y futura deberán ser constatados. El crecimiento ha favorecido especialmente a algunas de las CCAA, como Madrid y Extremadura. Todas las CCAA tienen un valor de IDG menor que su valor de IDH en los años estudiados, mostrando que cuando el desarrollo de género es considerado, las medidas de desarrollo humano disminuyen en las CCAA. Estos resultados confir- man el alcance de las desigualdades de género existentes en España en 1990 y 2000, y el espacio que queda por recorrer para conseguir una situación paritaria en el desarrollo ${ }^{17}$.

En cuanto a las limitaciones del presente estudio cabe destacar que, a partir del año 2000 no existe información sobre el IDG según CCAA, lo que podría estar indicando el desinterés por la medición del desarrollo de género. Algunas explicaciones llevan a pensar que el IDG sea una herramienta útil para medir desigualdades de género solo en países no desarrollados. En sociedades desarrolladas las necesidades de supervivencia y educación están cubiertas en gran medida, por lo que el componente de ingresos es el que se lleva todo el peso explicativo del IDG $^{23,24}$ para lo cual hay otros indicadores. Un aspecto importante sobre el índice de educación es el reparto de las proporciones para componerlo pues, por un lado, la tasa bruta de matriculación combinada es sólo una tercera parte del IDG, e incluye la educación primaria en la que difícilmente se encontraran diferencias, pues en España es obligatoria. Por otro lado, la tasa de alfabetización de adultos (2/3 partes del IDG) en España es similar entre ambos sexos. Por lo tanto, para hallar diferencias en la componente de educación del IDG entre hombres y mujeres en los países desarrollados sería conveniente otorgar más peso a la matriculación combinada pero sólo para la educación secundaria y terciaria.

Otra limitación del propio índice (IDG) es que no considera aspectos sociales relevantes en las desigualdades de género, como la participación en el mercado de trabajo, la segregación horizontal y vertical del trabajo, el tipo de contrato (temporales o sin contrato), y la participación en el trabajo reproductivo. La información sobre estos aspectos, en los que las mujeres están en desventaja respecto a los hombres, está contemplada en otros indicadores de des- 
igualdades de género como el IPG e IBG ${ }^{7,8}$, entre otros, pero no permiten un análisis interregional debido a que solo están disponibles a nivel de país.

Así como los indicadores de empoderamiento de las mujeres utilizan medidas absolutas donde lo que se mide es su desarrollo, del uso de medidas relativas como el IDG se deriva la dificultad de interpretar si la mejoría en el desarrollo de género se debe al descenso de los valores de los hombres manteniéndose constante los de las mujeres, por el incremento de ambos con diferente velocidad de cambio o por que mejoran los valores de las mujeres mientras que los de los hombres permanecen constantes.

Madrid y Extremadura destacan en su crecimiento proporcional del IDG entre los años 1990 y 2000, aunque Madrid partía de la primera posición en el desarrollo de género y Extremadura de la última. Esta situación podría deberse a que ambas CCAA dispusieran de Planes de Igualdad de Oportunidades para hombres y mujeres en los albores de este tipo de iniciativas en España. La Comunidad de Madrid aprobó el I Plan de Igualdad en 1989-1991, II Plan 1992-1995 y III Plan 1997-2005. Extremadura aprobó el I Plan de Igualdad de Oportunidades en 1991, vigente hasta Julio de 2000, fecha en la cual se aprobó el II Plan con vigencia en el trienio 2000-2003 25,26. Ya en el año 2000 la Comunidad de Madrid (IDG 0,940) estaba en desarrollo de género por encima de Canadá $(0,932)$ y EEUU (0,927), países que disponían de los índices más altos a nivel mundial en IDG. En cambio, Extremadura (IDG 0,856) en las mismas fechas seguía en similares condiciones que Eslovenia $(0,857)$ y Portugal $(0,858)^{27}$. No obstante, este desarrollo podría también estar relacionado con otras políticas como las económicas, las de justicia social, educativas y de salud, entre otras, que pueden haber incidido positivamente de forma directa o indirecta en el estatus social de las mujeres y en la esperanza de vida de los hombres, por mejora de los entornos, contextos o niveles en los que viven mujeres y hombres ${ }^{28}$.

En España la esperanza de vida aumentó en ambos sexos entre 1990 y 2000 . Aunque por una parte sigue siendo mayor en mujeres por otra el crecimiento ha beneficiado levemente más a los hombres en la década de los 90. En consecuencia, las diferencias en la esperanza de vida entre ambos sexos se han reducido. Desde los estudios de género este resultado se explica como fruto de los riesgos a que están expuestos hombres y mujeres en función de la adscripción o construcción de la identidad social de género ${ }^{29-31}$. También como fruto de los cambios en la organización de la estructura social, mediante la cual la posición y las actividades de hombres y mujeres se circunscriben a determinados ámbitos productivos y reproductivos, respectivamente, que influirán en el acceso a recursos y distribución de estos en ambos sexos ${ }^{32,33}$. La disminución en las diferencias en la esperanza vida entre hombres y mujeres se explica en la literatura científica por la disminución de la exposición a ciertos factores de riesgo en hombres, o por el cambio de exposición en las mujeres hacia los clásicamente asociados a la identidad masculina. Este es el caso de las diferencias en el hábito de fumar y el empleo no cualificado ${ }^{34}$. La teoría de la Emancipación de las mujeres ${ }^{35}$ propone que, debido a los roles cambiantes de género y la liberalización concomitante de las normas relacionadas con las conductas de las mujeres, han disminuido las diferencias entre ambos sexos en la exposición a los factores de riesgo de mortalidad y, en consecuencia, también lo han hecho las diferencias en la esperanza de vida ${ }^{36}$.

Por otra parte, la teoría de la Modernización ${ }^{35}$ de los roles de género postula que la interacción de roles tradicionales con los actuales roles de género ha influido en las tendencias de las desigualdades de género 
en la exposición a factores de riesgo. Esto significa que en las mujeres los roles de género contemporáneos (como conducir coches) interactúan con los tradicionales (como llevar a los hijos al colegio) por lo que, por una parte, podrían proteger la salud de las mujeres (como no beber o beber poco alcohol para poder realizar las tareas de cuidados antes mencionadas) pero también podrían aumentar el riesgo de sufrir accidentes de tráfico ${ }^{37}$.

En relación al componente "educación" del IDG, el cual suele indicar hacia donde se está dirigiendo la persona, se evidencia que en España, entre ambos sexos, existe más igualdad según este índice que según la esperanza de vida. Las desigualdades de género en la educación se acortaron en los años noventa, pues el nivel de educación de las mujeres aumentó el doble respecto a los hombres. La educación ha estado en la base del cambio que se ha producido en la trayectoria de las mujeres, el cual es observado en todas las CCAA en el año 2000 respecto a 1990. Probablemente este hecho está facilitando pasar desde el ámbito doméstico al sector público, aunque no necesariamente exista un paralelismo entre el tipo de ocupación de las mujeres y su educación. Véase la incongruencia entre los logros potenciales y los reales por la existencia de prácticas de exclusión directa e indirecta, como la discriminación en la contratación y promoción, el acoso en el lugar de trabajo y la no conciliación de la vida laboral y familiar ${ }^{35}$. La presencia de la mujer en la escuela y en las universidades ha sido una reivindicación (junto con la del mercado laboral) clásica del movimiento feminista mundial. Durante la segunda mitad del siglo XX la tendencia fue favorable. Aunque el incremento en los niveles de educación se produjo tanto en hombres como en mujeres, la literatura científica internacional coincide en que es en éstas en las que en la década de los 90 el incremento fue mayor ${ }^{37}$.

El nivel de acceso al empleo de las mujeres en España es aún uno de los más bajos de Europa ${ }^{17}$. El componente que mide la desigualdad de ingresos entre hombres y mujeres ha mejorado pues se evidencia que en el 2000 en España existe más igualdad en los ingresos entre ambos sexos que en 1990. El nivel de ingresos de las mujeres ha aumentado prácticamente el triple respecto al de los hombres. Estos resultados concuerdan con los de la Organización de las Naciones Unidas (ONU) cuando afirman que las mujeres están obteniendo gradualmente un mayor acceso al empleo remunerado a un nivel similar al de los hombres. Aunque también la ONU afirma que las mujeres tienen mayor probabilidad que los hombres de tener un trabajo no remunerado, hecho que dificultaría alcanzar el Objetivo de la Meta del Milenio para el 2015 de igualdad de género y empoderamiento de las mujeres ${ }^{4}$.

La tasa de actividad en el año 2000 en las mujeres aún era baja (42\%) en comparación con la de los hombres $(66 \%)^{38}$. Incluso en ese mismo año en algunas CCAA la tasa de actividad de las mujeres solo llegó al 34\% (Asturias y Castilla La Mancha), 37\% (Andalucía) y 38\% (La Rioja y Aragón) ${ }^{38}$. En el año 2004 las cifras tampoco habían cambiado en gran medida, ya que los hombres presentaban una tasa de actividad del $68 \%$ y las mujeres del $45 \%$. En este sentido, se ha constatado que la baja tasa de actividad de las mujeres genera desigualdades de género en salud, sobre todo si se le agrega las desigualdades de género dentro del mercado laboral, como las condiciones de empleo y la exposición a riesgos laborales ${ }^{39}$. La tasa de paro también es un componente a considerar al hablar del desarrollo humano relativo al género ya que, pese a haber mejorado sus cifras en el período de estudio, continuaron siendo superiores en las mujeres. En España, la tasa de paro de los hombres en el año 2000 llegaba al 9\% y en las mujeres al 20\%, destacando que en algunas CCAA, la tasa de paro de las mujeres llegó a 35\% (Extremadura) y 33\% (Andalucía $^{40}$. 
La posición de España a nivel mundial en cuanto a desigualdades en el desarrollo de género no es especialmente desfavorable en comparación con otros países europeos. Pero estas desigualdades existentes entre sus propias regiones, más profundas de hecho que sus desigualdades en el desarrollo humano global del país, alertan de la necesidad de considerarlas en los procesos de planificación de políticas públicas. En concreto, las evidencias muestran que siguen las diferencias en el desarrollo norte-sur en España, siendo las CCAA del sur y oeste las menos desarrolladas económicamente desde la perspectiva de género, como ya sucedía hace 30 años $^{24}$. No obstante, algunas CCAA del norte y del este de España como Asturias y Baleares a final de la década de los 90 han desacelerado su desarrollo de género, descendiendo en la igualdad respecto al valor global del país. Además mientras que en 1990 algo más de la mitad de la población $(51,5 \%)$ se situaba por encima del IDG promedio de España, en 2000 descendió al $46,3 \%$, entre otros hechos debido al incremento de la población de algunas CCAA de bajo IDG, como Andalucía, Canarias y Comunidad Valenciana y al descenso de la población de ciertas CCAA de alto IDG, como Cantabria y Castilla y León. La puesta en marcha de Planes de Igualdad a nivel nacional y en las CCAA implican el esfuerzo añadido de asegurar que toda planificación, ejecución y evaluación de políticas públicas en cualquier sector cuente con la perspectiva de género como requisito esencial para eliminar, desde la base, las desigualdades entre la población española.

\section{AGRADECIMIENTOS}

Este estudio ha sido parcialmente financiado por el Observatorio de Salud de las Mujeres. Ministerio de Sanidad y Consumo, España. La primera firmante del artículo también recibió una beca predoctoral de codesarrollo de la Fundación Bancaja y Universidad de Alicante. También queremos agradecer especialmente a Vicente Clemente Gómez por su valioso apoyo técnico.

\section{BIBLIOGRAFÍA}

1. Osberg K, Sharpe A. An Index of Economic Wellbeing for Selected OECD Countries. Rev Income \& Wealth 2002; 48: 291-316.

2. Organización de Naciones Unidas. Programa de las Naciones Unidas para el Desarrollo. Oficina de Desarrollo Humano. Informe Mundial de Desarro1lo Humano 1990. (Citado 18 de May. 2007). Disponible en: http://pnud.sc17.info/files/InfoMundiales/IDH\%201990.pdf.

3. Organización de Naciones Unidas. Programa de las Naciones Unidas para el Desarrollo. Oficina de Desarrollo Humano. Informe Mundial de Desarrollo Humano 1995. (Citado 18 de May. 2007). Disponible en: http://pnud.sc17.info/files/InfoMundiales/IDH\%201995.pdf

4. Organización de Naciones Unidas. Objetivos de Desarrollo del Milenio: Informe de 2007. New York 2007. Citado 25 de Sept. 2007. (Citado 25 de Sept. 2007) Disponible en: http://www.un.org/spanish/millenniumgoals/report2007/mdgreport2007r2.pdf

5. Naciones Unidas. Informe de la Cuarta Conferencia Mundial sobre la Mujer (Citado 01 de Oct. 2007). Disponible en: www.un.org/womenwatch/confer/beijing/reports/platesp.htm

6. Naciones Unidas Beijing+5. Período extraordinario de la Asamblea General. Mujeres 2000: Igualdad de género, desarrollo y paz para el siglo XXI. Nueva York: 2000 (Citado 01 de Oct. 2007). Disponible en: www.un.org/spanish/conferences/Beijing/mujer2021.htm

7. Hausmann R, Tyson LD, Zahidi S. World Economic Forum. The Global Gender Gap Report 2007 Ginebra. (Citado 30 de Nov. 2007). Disponible en: www.weforum.org/en/initiatives/gcp/Gender\%20Gap/index.htm

8. Organizaciones de Naciones Unidas. Programa de Naciones Unidas para el Desarrollo (PNUD) Informe sobre desarrollo humano 2000. New York 2000. (Citado 30 de Nov. 2007). Disponible en: http://pnud.sc17.info/files/InfoMundiales/IDH\%2 02000.pdf

9. Pollet A. Women, Work and Equal Opportunities in Post-communist Transition. Work, Employment and Society 2003; 17: 331-57. 
10. Lyness KS, Kropf MB. The relationships of national gender equality and organizational support with work-family balance: A study of European managers. Hum Relat 2005; 58: 33-60.

11. Metcalfe BD, Afanassieva M. Gender, work, and equal opportunities in central and eastern Europe. Women in Management Review 2005; 20: 397-411.

12. Yu M-Y, Sarry R. Women's Health Status and Gender Inequality in China. Soc Sci Med 1997; 45: 1885-98.

13. Vives-Cases C, Álvarez-Dardet C, Carrasco-Portiño M, Torrubiano-Domínguez J. El impacto de la desigualdad de género en la violencia del compañero íntimo en España. Gac Sanit. 2007; 21: 242-6.

14. Varkey S, Gupta S. How gender (in) sensitive are the gender-related indices? Bull World Health Organ 2005, 83 (12): 954-6.

15. Tortosa-Ausina E, Pérez F, Mas M. Growth and Convergence Profiles in the Spanish Provinces. J Regional Sci 2005; 45: 147-82.

16. Marchante A, Ortega B, Sánchez J. The Evolution of Well-being in Spain (1980-2001): A Regional Analysis. Soc Indic Res 2006; 76: 283-316.

17. Martínez Peinado J, Cairó Céspedes G. Gender and Regional Inequality in Human Development: The Case of Spain. Fem Econ. 2004; 10: 37-64.

18. Starfield, B. State of the art in research on equity in health. J Health Polit Policy Law 2006; 31(1): 11-32.

19. Kawachi I, Kennedy BP, Gupta V, Prothrow-Stith D. Women's status and the health of women and men: a view from the States. Soc Sci \& Med 1999; 48: 21-32.

20. Artazcoz L, Borrell C, Cortès I, Escribà-Agüir V, Cascant L. Los determinantes de la salud en una perspectiva integradora de los enfoques de género, clase social y trabajo. En: Borrell C, Artazcoz L, coordinadoras. 5 ${ }^{a}$ Monografía de la Sociedad Española de Epidemiología. Investigación en Género y Salud. Barcelona: Sociedad Española de Epidemiología; 2007. p. 21-43.

21. Herrero C, Soler A, Villar A, Sabater S (Ed). Capital Humano y Desarrollo Humano en España, sus Comunidades Autónomas y Provincias 19802000. Valencia: Instituto Valenciano de Investigaciones Económicas; 2004.

22. Bryman A, Cramer D. Quantitative data analysis with SPSS release 10 for Windows: a guide for social scientists. London: Editorial Routledge; 2001.

23. Bardhan K, Klasen S. UNDP's Gender-Related Indices: A Critical Review. World Development 1999; 6: 985-1010.

24. Goerlich F, Mas M. Inequality in Spain 19731991: Contribution to a Regional Database. Rev Income \& Wealth 2001; 47: 361-78.

25. Comunidad de Madrid. Área de Promoción de la Igualdad y Empleo. Dirección General de la Mujer. Planes y Actuaciones. Planes de Igualdad de Oportunidades entre Hombres y Mujeres. (Citado 02 de Oct.2007). Disponible en: http://www.madrid.org/ cs/Satellite $\mathrm{c}=$ Page \&cid=1109265843983\&idCons e je ri a $=1109266187284 \&$ id Lis tConsj $=1109265444710 \&$ pagename $=$ ComunidadMadrid\%2FEstructura\&pid=1109265444699\&sm= 1109265843983

26. Junta de Extremadura. Consejería de Cultura. Instituto de la Mujer Extremeña. Publicaciones. Planes de Igualdad de Oportunidades entre Hombres y Mujeres. (Citado 02 de Oct. 2007). Disponible en: http://www.mujerextremadura.com/publicaciones.htm

27. Organización de Naciones Unidas (ONU). Informe sobre el Desarrollo Humano 2000. (Citado 02 de Oct.2007). Disponible en: http://www.aidh.org/ViolDE/pdf_e/ch0.pdf

28. Krieger N. Proximal, Distal, and the Politics of Causation: What's Level Got to Do With It? Am J Public Health 2008; 98(2): 221-230.

29. Organización Panamericana de la Salud (OPS). Informe Género, Salud y Desarrollo en las Américas. Indicadores Básicos 2005. (Citado 01 de Nov.2007). Disponible en: http://www.paho.org/Spanish/AD/GE/GenderBrochureSP05.pdf

30. Ruiz Cantero MT, Papí Gálvez N, Cabrera Ruiz V, Ruiz Martínez A, Álvarez-Dardet Díaz C. Los sistemas de género y/en la Encuesta Nacional de Salud. Gac Sanit. 2006; 230: 427-34.

31. Arber, S. Comparing inequalities in women's and men's health in Britain in the 1990's. Soc Sci \& Med 1997: 44(6); 773-88.

32. Waldron I. Trends in gender differences in mortality: relationships to changing gender differences in behaviour and other causal factors. In: Annandale $\mathrm{E}$, Hunt $\mathrm{K}$, eds. Gender Inequalities in Health. Buckingham: Open University Press; 2001. p. 150-181. 
33. Álvarez-Dardet C, Montahud C, Ruiz MT. The widening social class gap of preventive health behaviours in Spain. Eur J Public Health 2001; 11: 225-6.

34. Annandale E, Hunt K. Gender inequalities in health (Edit). Buckingham: Open University Press; 2000.

35. Waldron I, Weiss CC, Hughes ME. Interacting effects of multiple roles on women's health. J Health Soc Behav. 1998; 39:216-36.

36. Mathews S, Hertzman C, Ostry A, Power C. Gender, work roles and psychosocial work characteristics as determinants of health. Soc Sci \& Med 1998;46 (11): 1417-24
37. Oakley A. Science, gender and women's liberation: an argument against postmodernism. Womens Stud Int Forum. 1998; 21(2): 133-46.

38. Universidad de Navarra. Business School (IESEIRCO). Euro índice Laboral Adecco. $\mathrm{N}^{\circ}$ 9: 1-8. (Citado 02 de Oct. 2007). Disponible en: http://www.iese.edu/es/RCC/IRCO/Investigacion/Docsypubl/Indices/Indices.asp

39. Artazcoz L, Escribá-Agüir V, Cortés I. Género, Trabajo y Salud en España. Gac Sanit. 2004; 18: 24-35.

40. Instituto Nacional de Estadística. INEBASE. Encuesta de Población Activa (metodología 2005) (Citado 30 de Nov. 2007). Disponible en: http://www.ine.es/jaxi/menu.do?type=pcaxis $\&$ pat $\mathrm{h}=/ \mathrm{t} 22 / \mathrm{e} 308 \_\mathrm{mnu} \&$ file $=$ inebase $\& \mathrm{~N}=\& \mathrm{~L}=0$ 
